\title{
The iLearnRW Game: Support for Students with Dyslexia in Class and at Home
}

\author{
Thomas Cuschieri, Rilla Khaled, Vincent E. Farrugia, Héctor P. Martínez, Georgios N. Yannakakis \\ Institute of Digital Games, University of Malta \\ Msida, Malta 2080 \\ Email: \{thomas.cuschieri, rilla.khaled, vincent.farrugia, hector.p.martinez, georgios.yannakakis\}@um.edu.mt
}

\begin{abstract}
Dyslexia includes a large variety of literacy-related difficulties which demands, in most cases, a personalised intervention. However, as dyslexia affects a large fraction of the population, schools cannot provide individual care for each student. The iLearnRW game provides a tool for students to work on their literacy skills following a personalised teaching programme. The design of the game and adaptation mechanisms integrated with it are aimed at maintaining student engagement for the duration of an open-ended number of playing sessions, while using a limited quantity of assets and literacy content. By focusing on maintenance of engagement, we hope to improve learning outcomes and motivate students to also play the game outside of school.
\end{abstract}

\section{INTRODUCTION}

Dyslexia is a very common learning disability, affecting over $10 \%$ of the population. Due to the limited resources available in schools, many dyslexic students do not receive the specialised education they require to overcome their learning issues, thus creating compound learning issues later in their educations. New software technologies present themselves as an alternative to traditional teaching, providing solutions that can identify and support students' reading deficits without the constant supervision of a teacher.

Within the different teaching strategies for literacy aimed at students with dyslexia, overlearning is always given a top priority. This technique consists of requiring the student to regularly repeat the same activities or exercises in order to acquire and maintain skills recently acquired, as this is viewed as contributing towards gains in speed and accuracy with regards to literacy skills. In light of how in games players are typically expected to learn core mechanics and apply them repeatedly, digital games offer considerable potential as part of dyslexia interventions. In addition, a well-designed and rich game can keep a student engaged while working on educational activities, and even motivate them to work on them outside schools.

As part of a larger educational software system for reading and writing (iLearnRW), we are currently developing a game to help students strengthen their literacy skills with or without teachers present ${ }^{1}$. While the idea of games for dyslexia is not new, we contribute novel game mechanics that facilitate ongoing and open-ended re-playability and adaptation mechanisms to support unsupervised effective learning. The design of the game has been guided by participatory design methods that

\footnotetext{
${ }^{1}$ The current prototype can be downloaded from http://www.um.edu.mt/digitalgames/gameresearch/ilearnrw/
}

have allowed both end users and dyslexia experts to contribute to the process, which have led to unique features not present in other games for dyslexia. At the adaptation end, we have combined an expert-driven student model with a crowd-sourced lesson planner, which integrates well-known structures about curriculums for literacy (long-term planning) with practical knowledge on lesson structuring in the classroom (short-term planning).

\section{GAme Design FeAtures}

Games for dyslexia often implement a collection of activities that are connected by a common visual theme and can be played independently of each other (e.g. WordShark [1], [2]). On the other hand, a smaller number of games are built around a rich narrative in which the student progresses through a story by solving language-related activities (e.g. Daisy Quest \& Daisy's Castle [3]). The iLearnRW game draws together these two alternatives and provides a common interface and metaphor for the visualisation of learning progress.

Rather than designing a narrative built around a fixed story, we decided to build a game around characters. While we could have opted to pursue a design featuring a partially adaptable narrative, because the overall narrative and core plot points would not have changed from one play-through to another, it would have resulted in a game offering low engagement value for players having completed the game once already. Instead, a game that engages players through interesting characters that co-exist within the same world automatically has further replayability value than games with relatively fixed narratives, as the game does not define a formal ending. While such a design does not guarantee infinite re-playability, it provides a simple solution for game extension. Instead of needing to build content into a game with a fixed beginning and ending, a designer can easily inject new characters loosely related to those already existing in the game world.

In tying together the two alternative modes of play and a visualisation for player progress, we have opted to present the player achievements through reference to characters from the game world. The game world features Día de los Muertostype characters. Each of the character represents a group of language difficulties that the student will practise every time she initiates an activity related to them. Following an initial interaction with a new character, the student earns that character as a friend, who is displayed on a social networklike interface. For example, when the user initiates a suffixing activity featuring Solomon, Solomon becomes her friend. As the student improves her skills with respect to a particular 
literacy difficulty, the relation with the corresponding character is reinforced and displayed in the social network. The friend list therefore serves as a summary of the learning difficulties a user has attempted to tackle and has potentially mastered within the system. In addition, the profiles of each of the characters serves as a direct link to activities related to specific difficulties, which allows a player uninterested on the game world (or directed by a teacher) to circumvent the need to navigate to these activities from within the game world.

Furthermore, by including a list of upcoming events within the social network, we create within the same metaphor a link from the social network back into the game world, fully connecting both modes of use within the same virtual world.

The available game activities consist of redesigned versions of nine literacy exercises defined by experts on dyslexia (e.g. segmentation of words or completion of sentences). The objective of this process is not only to adapt the exercises to the game theme but also to introduce more entertaining and engaging mechanics not directly related to literacy. For instance, the segmentation activity in which the student splits words by syllables one by one was transformed into "Solomon's Junkyard", in which blocks formed by the segmented syllables pile up at the bottom of the level, requiring the student to rearrange them before the level is completely filled (see Figure 1b); and the sentence-completion exercise becomes "Serenade Hero", where the player has to fill gaps in consecutive sentences with the right words on a limited time, in order to add more musicians to the serenader's band (see Figure 1a). Each of the activities will be also linked to specific locations in the world, defining a context for different stories as the same characters are met in different places (while guiding the student to work on the same difficulties through different activities).

\section{ADAPTATION MECHANISMS}

As dyslexia occurs on a continuum, the difficulties that affect students vary, not to mention that some students will learn faster than others or prefer playing sessions of different lengths. Therefore, to enable students to use the software without a personal teacher who dictates what to study next, the game needs to track student progress and adapt accordingly.

We build an adaptation mechanism based on two modules: a student model and a lesson planner. The student model contains a list of difficulties derived by dyslexia experts. For each difficulty, the model keeps track of the student's skill based on her performance during game activities and the time elapsed since the last practice. Furthermore, the list of difficulties is ordered based on dyslexia intervention programs used by teachers to structure their courses. While this ordering serves as general best practice for which difficulties are best mastered when, a linear presentation of the difficulties as the student masters them could be detrimental to engagement. Note that if the aforementioned approach was adopted in our game, in the case that a student could not improve a particular skill, she would be stuck in that part of the game, diminishing motivation to continue playing. This motivates the need of a lesson planner that balances the goals of advancing in the curriculum, reinforcing already acquired concepts, and keeping the student engaged and motivated (e.g. if the student is having difficulties learning the drop rule, then the system might inject

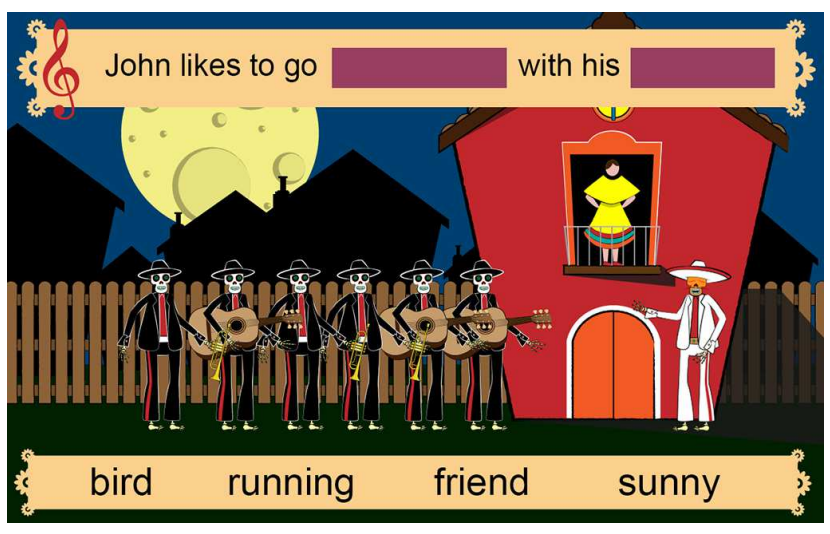

(a) Game activity example: Serenade hero

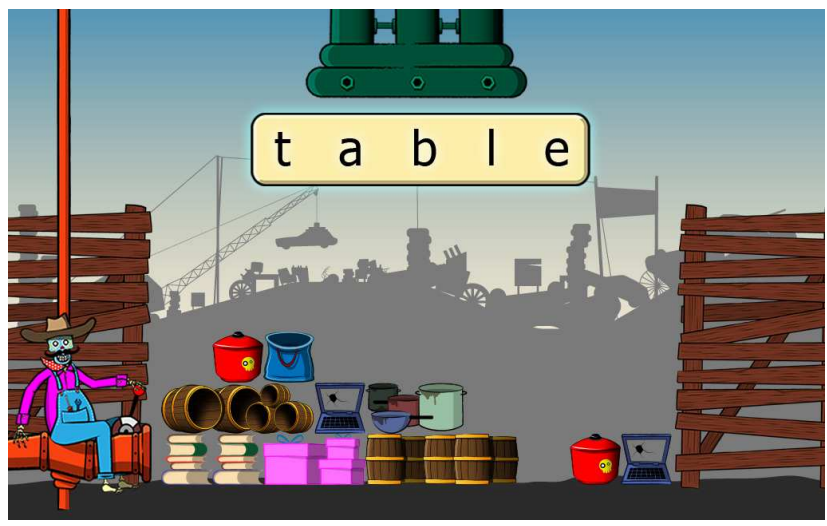

(b) Game activity example: Solomon's junkyard

Fig. 1. Screenshots of the iLearnRW game

activities for basic suffixing that the student already knows, thus increasing her morale). Since this knowledge is not documented, we are using crowd-sourcing and machine learning tools to learn the planner directly from simple simulated teaching sessions guided by dyslexia teachers.

These adaptation mechanisms are integrated seamlessly with the design described before. First, the student model is directly linked to the information displayed in the social network. Secondly, the lesson planner plots the next character encounters in the game world, and prepares the content that will be utilised in the game activities.

\section{ACKNOWLEDGEMENTS}

The authors would like to thank all the participants of the experiments. This research was supported, in part, by the ILearnRW (project no: 318803) FP7 ICT EU project.

\section{REFERENCES}

[1] C. Singleton and F. Simmons, "An evaluation of wordshark in the classroom," British journal of educational technology, vol. 32, no. 3, pp. 317-330, 2001

[2] L. Rello, C. Bayarri, and A. Gorriz, "What is wrong with this word? dyseggxia: a game for children with dyslexia," in Proceedings of the 14th international ACM SIGACCESS conference on Computers and accessibility. ACM, 2012, pp. 219-220.

[3] T. A. Barker and J. K. Torgesen, "An evaluation of computer-assisted instruction in phonological awareness with below average readers," Journal of Educational Computing Research, vol. 13, no. 1, pp. 89-103, 1995. 